\title{
Stage IA Gastric Gastrointestinal Stromal Tumor
}

National Cancer Institute

\section{Source}

National Cancer Institute. Stage IA Gastric Gastrointestinal Stromal Tumor. NCI

Thesaurus. Code C87825.

Stage IA includes: T1 or T2, N0, M0, Mitotic rate: Low. T1: Tumor $2 \mathrm{~cm}$ or less in greatest dimension. T2: Tumor more than $2 \mathrm{~cm}$, but not more than $5 \mathrm{~cm}$, in greatest dimension. N0: No regional lymph node metastasis. M0: No distant metastasis. (AJCC 7th ed.) 